\title{
A deficiência visual e o processo de construção da cidadania: um estudo no Instituto de Cegos do Brasil Central de Uberaba
}

\author{
The visual impairment and the process of construction of \\ citizenship: a study in the Institute for the Blind of Brasil Central \\ of Uberaba, Minas Gerais, Brazil
}

\author{
La debilitación visual y lo proceso de construcción de la \\ ciudadanía: un estudio en el Instituto para Ciegos del Brasil \\ Central de Uberaba, Minas Gerais, Brasil
}

Recebido: $19 / 08 / 2013$

Aprovado: 02/11/2013
Claudia Helena Julião ${ }^{1}$

Marlene Aparecida Manzan Paiva² Suraya Gomes Novais Shimano ${ }^{3}$

Verena Conti ${ }^{4}$

Zilda Cristina dos Santos 5

Esta pesquisa teve como objetivo conhecer a realidade cotidiana dos deficientes visuais atendidos no Instituto de Cegos do Brasil Central de Uberaba-ICBC, analisando qual é o impacto que as atividades socioassistenciais, de habilitação e reabilitação desenvolvidas na referida instituição causam no processo de construção da cidadania dessas pessoas. Para tanto, utilizou-se a pesquisa documental e de campo, com a realização de entrevistas com cinco deficientes visuais que vivem na instituição em regime de internato e 10 que são atendidos em regime de externato. Os resultados demonstraram que as ações desenvolvidas possibilitam a inserção do deficiente visual na sociedade, melhoria da qualidade de vida, favorecendo o processo de construção da cidadania e conquista da autonomia.

Descritores: Autonomia pessoal; Pessoas com deficiência visual; Serviço Social.

This research aimed to meet the everyday reality of the visually impaired attended at Institute for the blind of Central Brazil to Uberaba-ICBC, Minas Gerais, Brazil, analyzing what is the impact that social assistance activities and enabling and rehabilitation developed at the institution in the process of causing the construction of citizenship of these people. To this end, it used the documentary research and field, with qualitative approach and interviews with five visually impaired living in boarding institution and 10 that are serviced in extern scheme. The results demonstrated that the actions developed allow the insertion of the visually impaired into society, improving the quality of life, promoting the process of the construction of citizenship and achievement of autonomy.

Descriptors: Personal autonomy; People with visual impairments; Social Service.

Esta investigación tuve como objetivo conocer la realidad cotidiana de las personas con discapacidades visuales asistidos por el Instituto para Ciegos del Brasil Central de Uberaba-ICBC, Minas Gerais, Brasil, analizando cuál es el impacto que las actividades socioassistenciales, de habilitación y rehabilitación desarrolladas en la institución causan en la construcción de la ciudadanía de estas personas. Con este fin, utiliza el campo y la investigación documental, con enfoque cualitativo a traves de entrevistas a cinco invidentes viviendo en la institución y 10 que son atendidos en régimen de externado. Los resultados demostraron que las acciones desarrolladas permiten la inserción de los discapacitados visuales en la sociedad, mejorando la calidad de vida, promoviendo el proceso de la construcción de ciudadanía y el logro de la autonomía.

Descriptores: Autonomía personal; Personas con discapacidad visual; Servicio Social.

\footnotetext{
${ }^{1}$ Assistente Social. Doutora em Serviço Social. Professora Adjunta do Departamento de Serviço Social da Universidade Federal do Triângulo Mineiro (UFTM). claudiahj@servicosocial.uftm.edu.br.br

${ }^{2}$ Graduada do curso de Serviço Social da UFTM. marlene.mzn@gmail.com

${ }^{3}$ Fisioterapeuta. Doutora em Ciências da Reabilitação. Professora Adjunta do Departamento de Fisioterapia da UFTM. surayagnovais@gmail.com

${ }^{4}$ Assistente Social. Especialista em Docência do Ensino Superior. Assistente Social do Hospital das Clínicas da UFTM (HCUFTM). Assistente Social do Instituto dos Cegos do Brasil Central (ICBC). verenaconti@hotmail.com

${ }^{5}$ Assistente Social. Especialista em Saúde do Adulto. Assistente Social HC-UFTM. Assistente Social do ICBC. zilda.cristina.ss@gmail.com
} 


\section{INTRODUÇÃo}

A realização deste estudo foi motivada pela preocupação quanto às questões que envolvem a deficiência visual e as implicações que estas podem trazer para o exercício da cidadania e a conquista da autonomia da pessoa com essa deficiência.

Tais indagações surgiram em decorrência do contato com diversos deficientes visuais atendidos no Instituto de Cegos do Brasil Central (ICBC), sendo tal experiência marcada por momentos repletos de significados. Esses deficientes visuais demonstravam muitas habilidades e potencialidades que, supostamente, teriam certo grau de dificuldade para ser desenvolvidas por uma pessoa cega ou com baixa visão.

Sabe-se que a visão é responsável por fornecer informações para organização sensorial, compreender o mundo ao redor e dar significado aos objetos, conceitos e ideias. Portanto, a perda da capacidade visual implica a limitação das informações e conhecimentos que favorecem o desenvolvimento motor, perceptivo e emocional, causando adversidades de natureza individual e coletiva, que afetam a qualidade de vida, as restrições ocupacionais e a autoestima. Esses fatores podem, ainda, limitar o exercício da cidadania.

Ao longo da história, ocorreram modificações na percepção social e política no que diz respeito à questão da deficiência. Os estudos de Figueira ${ }^{1}$ revelam que a deficiência esteve associada às crendices e superstições e, por séculos, a pessoa com deficiência foi considerada um "peso" para as suas famílias.

Um marco relevante para o movimento de luta pelos direitos das pessoas com deficiência foi o ano de 1981, declarado pela ONU como "Ano Internacional da Pessoa com Deficiência". Desde então, as pessoas com deficiência passaram a tomar consciência de si como cidadãs, a se organizar politicamente e a conquistar visibilidade na sociedade, com avanços expressivos para o segmento.
Entretanto, nas últimas décadas, esse processo tem sido conquistado de forma heterogênea, não linear, por meio de trajetórias singulares com avanços e retrocessos.

Quanto à definição de deficiência, Ribas $^{2}$ afirma que é algo quase impossível, pois explicar onde começam os limites de uma pessoa e aonde chegam os seus alcances é uma tarefa impalpável.

A Organização Mundial de Saúde também não consegue definir deficiência devido à complexidade do conceito, por isso criou a Classificação Internacional de Deficiências, Incapacidades e Desvantagens (CIDID) que não define, mas classifica a deficiência com um referencial padronizado, estabelecido com objetividade, abrangência $\mathrm{e}$ intensidades, relativizando o conceito e inserindo-o no contexto social, mostrando que a deficiência é um acometimento que pode sobrevir em crianças e adultos, homens e mulheres, brancos e negros, pobres e ricos, orientais e ocidentais ${ }^{3}$.

Na legislação brasileira, o Decreto n. ${ }^{\mathrm{o}}$ $5.296 / 2004^{4}$ apresenta os conceitos específicos dos diferentes tipos de deficiência, que podem ocorrer em diferentes áreas do desenvolvimento, como, por exemplo, a mental, da linguagem, da visão, e outras. Os conceitos apresentados nesse Decreto, apesar de algum avanço legal, não se sustentam em uma visão sociocultural, apenas na visão médica.

$\mathrm{Na}$ Política Nacional de Saúde da Pessoa Portadora de Deficiência, a deficiência visual é apontada como uma condição irreversível de diminuição da resposta visual, em decorrência de causas congênitas, hereditárias e adquiridas, mesmo após tratamento clínico e/ou cirúrgico e uso de óculos convencionais ${ }^{5}$. Essa diminuição pode ser leve, moderada ou severa e compõe o quadro de baixa visão e ausência total de visão (cegueira).

Atualmente é possível verificar significativos avanços na trajetória histórica das práticas e políticas sociais de atendimento às pessoas com deficiência. 0 
que não significa afirmar que essas conquistas tenham sido gratuitas, e muito menos que sejam satisfatórias. Pelo contrário, muito ainda há que ser mudado, como, por exemplo, o preconceito referente à pessoa com deficiência que ainda persevera em nossa sociedade; as leis de inclusão desse segmento populacional no mercado de trabalho, que ainda não são plenamente efetivas; a política de assistência social, que além de seletiva é também excludente; a inabilidade das escolas de ensino regular para receber crianças com deficiência, apesar de sua inclusão ser garantida pela Lei de Diretrizes e Bases da Educação- LDB; a luta pela eliminação de barreiras, que vão desde a adaptação de ambientes físicos até a transformação da mentalidade das pessoas, incluindo a própria pessoa com deficiência, para que esta possa ter acesso assegurado a todos os espaços, às informações, aos bens e serviços, e consequentemente, a efetivação de seus direitos enquanto cidadã 6 .

Dentro deste contexto, este estudo tem como objetivo conhecer a realidade cotidiana dos deficientes visuais atendidos no Instituto de Cegos do Brasil Central de Uberaba-ICBC, analisando qual é o impacto que as atividades socioassistenciais, de habilitação e reabilitação desenvolvidas na referida instituição causam no processo de construção da cidadania dessas pessoas.

\section{MÉTODO}

O ICBC, cenário desta pesquisa, é uma associação civil sem fins lucrativos, filantrópica, beneficente, reconhecida por sua utilidade pública municipal e federal, destinada à assistência social, saúde e educação de deficientes visuais, situada em Uberaba-MG. Foi fundada em 25 de agosto de 1942 e, além do atendimento direto ao deficiente visual, assiste às famílias dos usuários, com o propósito de garantir o acesso às políticas públicas, o fortalecimento das relações familiares e o rompimento com o preconceito e a vulnerabilidade social.

Para o atendimento a esse seguimento populacional, o ICBC conta com uma equipe composta por profissionais contratados, cedidos por órgãos públicos e voluntários que prestam serviços para seus usuários em regime de internato, semiinternato e externato.

A pesquisa foi realizada na perspectiva do materialismo histórico dialético que tem como fundamento a concepção dinâmica da realidade em sua totalidade, considerando a historicidade do sujeito como um fator relevante ao abordar os mais diversificados fenômenos, pois, conforme Gil ${ }^{7}$, os fatos sociais não podem ser entendidos isoladamente, abstraídos das influências políticas, econômica e culturais.

A pesquisa foi orientada pela abordagem qualitativa e, foi aprovada pelo Comitê de Ética da Universidade Federal do Triângulo Mineiro (UFTM). No momento da pesquisa os entrevistados ou seus responsáveis assinaram o Termo de Consentimento Livre e Esclarecido.

Para a pesquisa de campo, foram escolhidos 15 sujeitos por amostra intencional por ser a que se apresenta como uma representação do universo. Realizou-se a entrevista semiestruturada com cinco sujeitos selecionados dentre os deficientes visuais que vivem no ICBC em regime de internato, matriculados em escolas de ensino regular, e dez sujeitos selecionados dentre os que recebem atendimento no ICBC e foram atendidos pelo Serviço Social no período de março a julho de 2011, originando encaminhamentos para a equipe multiprofissional.

Os depoimentos foram gravados em equipamento MP4, e posteriormente foi feita a transcrição de todas as gravações, preservando-se na íntegra a fala dos sujeitos.

Após a coleta de dados, para melhor compreensão do material produzido, foi utilizada a análise do conteúdo, que, conforme Richardson ${ }^{8}$ é um método que pela sua cientificidade exige eficácia e rigor na compreensão de um discurso, fundamentando-se em teorias que expliquem as descobertas do pesquisador, bem como a transmissão da leitura dessa descoberta. 
A análise qualitativa deste estudo foi orientada por um posicionamento mediado pelo que expressam as palavras dos entrevistados e à busca da apreensão do contexto histórico, social, econômico e político determinante das vozes por eles produzidas.

Para a apresentação dos dados, os sujeitos foram identificados com nome fictícios, utilizando-se nome de flores, visando assim preservar a identidade dos mesmos.

\section{RESULTADOS}

Foram entrevistados 15 sujeitos no ICBC, sendo cinco que vivem em sistema de internato representados no Quadro $1 \mathrm{e}$, dez em sistema de externato, representados no Quadro 2, fazendo menção à idade, ao tempo em que se encontram em atendimento, à patologia, ao tipo de deficiência e ao estado civil.

Quadro 1. Entrevistados que vivem no internato do ICBC. Maio a Julho de 2011.

\begin{tabular}{|c|l|l|c|c|c|}
\hline Nome & Deficiência & \multicolumn{1}{|c|}{ Patologia } & Idade & $\begin{array}{c}\text { Tempo de } \\
\text { Internato } \\
\text { (anos) }\end{array}$ & Estado Civil \\
\hline Rosa & Cega & Retinopatia da prematuridade & 26 & 18 & Solteira \\
\hline Orquídea & Cega & Glaucoma congênito & 33 & 15 & Solteira \\
\hline Magnólia & Cega & Amaurose congênita de Leber & 18 & 12 & Solteira \\
\hline Crisântemo & Cego & Retinopatia & 54 & 11 & Solteiro \\
\hline Hortência & Baixa visão & Retinose pigmentar & 31 & 07 & Solteira \\
\hline
\end{tabular}

Fonte:ICBC

Quadro 2. Entrevistados atendidos no ICBC em regime de externato. Maio a Julho de 2011.

\begin{tabular}{|c|c|c|c|c|c|}
\hline Nome & Deficiência & Patologia & Idade & $\begin{array}{c}\text { Tempo de } \\
\text { Atendimento } \\
\text { ICBC (anos) }\end{array}$ & Estado Civil \\
\hline Jasmim & Cego & Tumor cerebral & 31 & 23 & Solteiro \\
\hline Cravo & Cego & Descolamento de retina & 22 & 17 & Solteiro \\
\hline Tulipa & Baixa visão & Toxoplasmose & 25 & 11 & Solteiro \\
\hline Girassol & Cego & Retinopatia da prematuridade & 19 & 11 & Solteiro \\
\hline Lírio & Cego & Atrofia do nervo óptico & 24 & 11 & Solteiro \\
\hline Dália & Baixa visão & Atrofia do nervo óptico & 84 & 09 & Solteira \\
\hline Miosótis & Baixa visão & Glaucoma & 42 & 03 & Solteiro \\
\hline Azaléia & Baixa visão & Tumor na hipófise & 38 & 02 & Solteira \\
\hline
\end{tabular}

Fonte:ICBC

Observa-se que o ICBC atende não só deficientes visuais, mas também aqueles com baixa visão (seis apresentam baixa visão e nove são cegos). Todos os pesquisados são solteiros, adultos, com o tempo de dois a 23 anos de internação ou acompanhamento pela instituição e, na sua maioria procedente de Uberaba e, com patologias de base variáveis. Dos 15 sujeitos entrevistados, em dois casos a origem da

\section{DISCUSSÃO}

A visão é um canal sensorial muito importante para a obtenção de informações e sua redução ou falta traz consequências para o desenvolvimento e a aprendizagem, deficiência foi adquirida e 13 hereditários congênitos.

Quanto ao perfil dos deficientes visuais atendidos no ICBC, identificou-se que a maioria da população atendida está na faixa etária entre 0 a 39 anos. Entre os atendidos em regime de internato, a maioria é composta por mulheres, enquanto no regime de externato predominam os homens.

exigindo, assim, que as pessoas com deficiência visual recebam cuidados especiais com base em estímulos sensoriais compensatórios, por outras vias perceptivas para o desenvolvimento das atividades diárias e de aprendizagem. 
Nesse sentido, cabe destacar que a maioria dos deficientes visuais que procura pelos atendimentos do ICBC apresenta dificuldades ou incapacidades para desenvolver as rotinas da vida cotidiana. $\mathrm{Na}$ maioria das vezes, essa dificuldade acarretabaixa estima e limitações para adaptação às atividades desenvolvidas na instituição, bem como em sua vida cotidiana, justificando, assim, o longo período de adaptação à sua condição e consequente permanência em atendimento no ICBC.

Quanto aos deficientes visuais que vivem no ICBC em regime de internato, além dessa conjuntura complexa apresentada, ainda há casos em que os vínculos familiares não existem mais. Foram institucionalizados quando ainda eram crianças ou adolescentes e, embora a instituição desenvolva ações na tentativa de resgatar esses vínculos, em alguns casos não se tem mais a localização dos familiares.

Todos entrevistados declararam 0 estado civil como solteiro. Esse dado pode mostrar que, apesar de a instituição promover atividades conjuntas que fortalecem a socialização e a formação de vínculos, há um isolamento social dos deficientes visuais. A limitação da visão pode influenciar de forma negativa as habilidades sociais e as relações interpessoais iniciais, devido à dificuldade que o deficiente visual tem de processar as informações imprescindíveis às interações sociais e às relações sociais com seus pares. Habilidades sociais naturais para os videntes como o sorriso, por exemplo, são um sinal visual para promover uma interação, enquanto, para os deficientes visuais, o sorriso não é uma resposta social natural.

Conforme aponta Gil9, devido à existência de riscos reais, a família, os amigos e demais pessoas das relações do deficiente visual tendem a superprotegê-lo desde criança. Com isso, naturalmente, o seu campo de sociabilidade acaba sendo restrito e, quando se torna adulto, pode se sentir inseguro diante de novos relacionamentos e novos ambientes, o que faz com que apresente de forma negativa o sentimento do medo de se relacionar com o outro.

Outro aspecto a ser destacado quanto à afetividade dos deficientes visuais, diz respeito à educação sexual. Se, por um lado, muitas vezes, estes são alvo de superproteção, por outro, sofrem com preconceitos e mitos - com imagens projetadas de pessoas assexuadas, incapazes e dependentes. Contudo, é essencial proporcionar a oportunidade para que os deficientes visuais possam expor suas dúvidas e receber informações verdadeiras e claras para concretizar a sua vivência afetiva e a sexualidade de forma tranquila $e$ responsável.

A deficiência visual traz consigo limitações ou impedimentos no que se refere à apreensão de conceitos, acesso à escrita, a orientação e mobilidade autônoma, às interações sociais e ao controle espacial. Essa dificuldade de mobilidade e locomoção é expressa na fala dos entrevistados:

[...] só em andar, porque eu caio muito, e eu [...] num ando direito. (Dália)

Vale ressaltar que a dificuldade de caminhar apresentada pelas pessoas com deficiência visual está condicionada pela limitação sensorial, mas também pelo meio em que vive. Diversos obstáculos encontrados nas vias públicas, como bancas de jornal, lixeiras e calçadas mal conservadas formam barreiras que prejudicam a acessibilidade dessas pessoas.

Embora o acesso físico das pessoas com deficiência nas cidades brasileiras seja amplamente garantido pela legislação, ainda é necessário conforme Nogueira ${ }^{10}$, o desenvolvimento de políticas públicas, principalmente municipais, para favorecer a acessibilidade das pessoas com deficiência ou mobilidade reduzida e assim, garantir o direito constitucional de ir e vir a todos os cidadãos sem nenhuma distinção.

Sobre a renda per capita familiar e a procedência da fonte dessa renda, verificouse que dos 15 sujeitos entrevistados, 11 recebem o Benefício de Prestação 
Continuada (BPC), três são aposentados por invalidez e um é mantido pela família. Constata-se, desta forma que a maioria da população atendida no ICBC é composta por famílias de baixa renda que necessitam de proteção social para satisfazer suas necessidades básicas.

Os deficientes visuais que recebem o BPC avaliam tal benefício sob três perspectivas. A primeira, como um mecanismo de segurança e renda garantindo o consumo de bens básicos, conforme indica o depoimento a seguir:

Ajuda muito dentro de casa. Metade do meu dinheiro vai para comprar coisas pra dentro de casa. Arroz, feijão. (Narciso)

$\mathrm{Na}$ segunda perspectiva, o BPC é apontado como um instrumento de proteção básica para proteger os deficientes e suas famílias da situação de vulnerabilidade social em decorrência do desemprego, da pobreza e do emprego informal; e a terceira, como uma possibilidade para aumentar a independência social dos deficientes e de suas famílias, para que estes não fiquem a vida toda na dependência do benefício.

Verifica-se, de modo geral, que os entrevistados fazem uma avaliação positiva do BPC, dados que também são indicados em outros estudos 11 .

Nesse contexto, apesar de o BPC se configurar como um benefício com critérios de elegibilidade e repasse de valores seletivos, foi criado como uma política social integrante da rede de proteção social, baseado em políticas públicas onde o Estado é o responsável por atender as demandas e garantir a cidadania, diminuindo a vulnerabilidade social.

0 grau de escolaridade entre os deficientes visuais no ICBC é baixo, sendo que a maioria não concluiu o ensino fundamental. Embora nas últimas décadas tenha-se registrado considerável avanço na conquista da igualdade e do exercício dos direitos da pessoa com deficiência, o acesso e permanência da pessoa com deficiência visual na escola ainda são escassos.
Neste sentido, a inclusão das pessoas com deficiência no ensino regular, conforme dispõe a LDB, vem caminhando num processo de ordenamento, e em um conjunto de ações de políticas públicas. As ações do Estado não correspondem à demanda existente, e apenas uma pequena parcela dessa população é atendida. Além disso, existe uma grande dificuldade para a ampliação dos serviços de atendimento aos deficientes visuais devido à carência de professores especializados e à insuficiência de materiais para estudos e pesquisas, tanto em Braile como em ampliações para os alunos com baixa visão.

No que diz respeito à percepção dos sujeitos quanto aos serviços socioassistenciais prestados pelo ICBC, verifica-se que os mesmos tem boa aceitação entre os entrevistados conforme se vê nos relatos a seguir:

Eu avalio que é uma coisa boa, é um preparo ótimo para o deficiente [...] é sensacional. (Cravo)

São bons, porque ajudam a melhorar o meu desempenho, a minha mobilidade (Amor Perfeito)

Me ensinou a andar, me ensinou a conversar com as pessoas, a me comportar nos lugares. (Narciso)

Os sujeitos reconhecem que os serviços oferecidos pelo ICBC contribuem para a melhora no desenvolvimento, na mobilidade, nas relações sociais, na autoestima, de modo que consigam lidar da melhor forma possível com as limitações acarretadas pela deficiência.

\section{CONCLUSÃO}

As pessoas com deficiência viveram durante séculos por trás dos muros das casas de suas famílias, a quem cabia exclusivamente a responsabilidade sobre elas. Foi uma longa trajetória até que as questões envolvendo as pessoas com deficiência passassem a ser vistas como uma questão de política pública, superando os mecanismos de exclusão, assistencialismo e caridade que foram 
edificados culturalmente em nossa sociedade.

As principais conquistas para as pessoas com deficiência visual aconteceram após os movimentos sociais dos 1980, que asseguram vários direitos para esse segmento populacional em vários aspectos e setores como, por exemplo, na saúde, na educação, na inserção no mercado de trabalho, na inclusão social, e outros.

Diversas e diferentes barreiras afetam cotidianamente as pessoas com deficiência. As barreiras físicas são aquelas que limitam ou impedem o acesso, a liberdade de movimento e a circulação com segurança. As barreiras sistêmicas são as relacionadas às políticas formais e informais, como, por exemplo, escolas e estabelecimentos que não oferecem atendimento adequado para as pessoas com deficiência. Além disto, há as barreiras atitudinais, que são os preconceitos, estigmas e estereótipos que implicam comportamentos negativos como o repúdio, a discriminação e a exclusão baseados na condição física, mental ou sensorial.

Em relação à pessoa com deficiência visual, a superação dessas barreiras envolve oferecer-lhe as mesmas possibilidades que um vidente para desenvolver e exercer sua cidadania, seus direitos e deveres no campo social, econômico, político e profissional.

É importante os programas de habilitação e reabilitação visual, como o oferecido pelo ICBC, os quais possibilitam diagnosticar, tratar e acompanhar o deficiente visual no desenvolvimento funcional da visão e estimular sua independência nas atividades cotidianas. Verifica-se que os serviços socioassistenciais do ICBC possibilitam melhorar a qualidade de vida, desenvolver habilidades e talentos, favorecer o processo de construção da cidadania e a conquista da autonomia dos deficientes visuais e de suas famílias, por meio dos serviços prestados por uma equipe multiprofissional.

É preciso que a sociedade como um todo também esteja disposta a participar desse processo, de forma a atender na totalidade a especificidade de cada deficiente visual, superando as atitudes de preconceito e discriminação e contribuindo para a conquista da autonomia e de condições que propiciem aos deficientes assumir o papel de cidadãos.

\section{REFERÊNCIAS}

1. Figueira E. Caminhando em silêncio: uma introdução à trajetória das pessoas com deficiência na história do Brasil. São Paulo: Giz; 2008.

2. Ribas JBC. Preconceito contra pessoas com deficiência: as relações que travamos com o mundo. São Paulo: Cortez; 2007.

3. Organização Pan-Americana da Saúde, Organização Mundial da Saúde [site da Internet]. Brasília: OPAS; OMS; [atualizado em 2010; citado em 18 set 2011]. Disponível em: http://new.paho.org/bra

4. Brasil. Decreto ${ }^{\circ} 5.296$ de 02 de dezembro de 2004. Regulamenta as Leis nos 10.048, de 8 de novembro de 2000, que dá prioridade de atendimento às pessoas que especifica, e 10.098, de 19 de dezembro de 2000, que estabelece normas gerais e critérios básicos para a promoção da acessibilidade das pessoas portadoras de deficiência ou com mobilidade reduzida, e dá outras providências [Internet]. D.O.U. 3 dez 2004 [citado em 14 fev 2013]. Disponível em: http//www.cedipod.org.br

5. Brasil. Decreto no 3.298 de 20 de dezembro de 1999. Regulamenta a Lei no 8.753 de 24 de outubro de 1989, dispõe sobre a Política Nacional para a Integração da Pessoa Portadora de Deficiência, consolida as normas de proteção, e dá outras providências [Internet]. D.O.U. $21 \mathrm{dez}$ 1999 [citado em 14 fev 2013]. Disponível em: http://www.planalto.gov.br/ccivil_03/decreto/d 3298.htm.

6. Ministério da Educação (Br). Lei 9.394 de 20 de dezembro de 1996. Estabelece as diretrizes e bases da educação nacional [Internet]. D.O.U. 23 dez. 1996 [citado em 01 out. 2011]. Disponível em:

http://www.planalto.gov.br/ccivil_03/leis/1939 4.htm

7. Gil AC. Métodos e técnicas de pesquisa social. 6 ed. São Paulo: Atlas; 2009.

8. Richardson RJ. Pesquisa social: métodos e técnicas. 3 ed. São Paulo: Atlas; 2011. 
9. Gil M, org. Deficiência visual [Internet]. Brasília: Ministério da Educação. Secretaria de Educação à Distância. 2000 [citado em $14 \mathrm{fev}$ 2013]. Disponível em: www.educacao.salvador.ba.gov.br.

10. Nogueira G. Acessibilidade?...A lei garante [Internet]. [citado em $10 \mathrm{fev}$ 2013]. Disponível em: http://www.crfaster.com.br/cc22.pdf

11. Sochaczevski J, Tavares LHGL. SUAS, BPC e pessoas com deficiência: análise a partir de municípios fluminenses. Ser Social 2013; 15(32):141-65.

\section{CONTRIBUIÇÕES}

Marlene Aparecida Manzan Paiva, Verena

Conti Zilda Cristina dos Santos contribuíram no levantamento bibliográfico, coleta de dados, análise dos dados e redação;

Suraya Gomes Novais Shimano e Claudia Helena Julião participaram no planejamento, delineamento do estudo e revisão crítica. 